\title{
Evaluation of Task fMRI Decoding With Deep Learning on a Small Sample Dataset
}

\author{
Sunao Yotsutsuji ${ }^{*}$, Miaomei Lei $^{2}$ and Hiroyuki Akama ${ }^{1,3}$ \\ ${ }^{1}$ School of Life Science and Technology, Tokyo Institute of Technology, Tokyo, Japan, ${ }^{2}$ Ex-Graduate School of Science \\ and Technology, Tokyo Institute of Technology, Tokyo, Japan, ${ }^{3}$ Institute of Liberal Arts, Tokyo Institute of Technology, Tokyo, \\ Japan
}

OPEN ACCESS

Edited by:

Rong Chen,

University of Maryland, Baltimore,

United States

Reviewed by:

Qinglin Dong,

Harvard Medical School,

United States

Xiang Li,

National Supercomputer Center.

China

*Correspondence:

Sunao Yotsutsuji

yotsutsuji.s.aa@m.titech.ac.jp

Received: 29 June 2020 Accepted: 25 January 2021

Published: 12 February 2021

Citation:

Yotsutsuji S, Lei M and Akama $H$

(2021) Evaluation of Task fMRI Decoding With Deep Learning on

a Small Sample Dataset.

Front. Neuroinform. 15:577451. doi: 10.3389/fninf.2021.577451
Recently, several deep learning methods have been applied to decoding in task-related $\mathrm{fMRI}$, and their advantages have been exploited in a variety of ways. However, this paradigm is sometimes problematic, due to the difficulty of applying deep learning to high-dimensional data and small sample size conditions. The difficulties in gathering a large amount of data to develop predictive machine learning models with multiple layers from fMRI experiments with complicated designs and tasks are well-recognized. Group-level, multi-voxel pattern analysis with small sample sizes results in low statistical power and large accuracy evaluation errors; failure in such instances is ascribed to the individual variability that risks information leakage, a particular issue when dealing with a limited number of subjects. In this study, using a small-size fMRI dataset evaluating bilingual language switch in a property generation task, we evaluated the relative fit of different deep learning models, incorporating moderate split methods to control the amount of information leakage. Our results indicated that using the session shuffle split as the data folding method, along with the multichannel 2D convolutional neural network (M2DCNN) classifier, recorded the best authentic classification accuracy, which outperformed the efficiency of 3D convolutional neural network (3DCNN). In this manuscript, we discuss the tolerability of within-subject or within-session information leakage, of which the impact is generally considered small but complex and essentially unknown; this requires clarification in future studies.

Keywords: brain decoding, cross-subject modeling, cross-validation, deep learning, fMRI, model selection, MVPA

\section{INTRODUCTION}

In cognitive neuroscience, the framework for predicting the stimuli given to subjects or the tasks they perform based on their neural activity is called "decoding." From a modeling perspective, we can evaluate predictive power and identify the brain regions that are the most informative for specific stimuli or tasks. Decoding has also been studied extensively in the context of mind-reading.

Abbreviations: 3DCNN, three-dimensional convolutional neural network; ANOVA, analysis of variance; CV, crossvalidation; FWHM, full width at half maximum; Leave One Subject Out, leave-one-subject-out cross-validation; M2DCNN, multichannel two-dimensional convolutional neural network; MNI, Montreal Neurological Institute; MVPA, multi voxel pattern analysis; Permutation, permutation test; PLR, penalized logistic regression; SVM, support vector machine; Test, test set; Train, training set; Valid, validation set. 
The most widely used decoding strategy is a pattern classification method called Multi Voxel Pattern Analysis (MVPA; Cohen et al., 2017). Haxby et al. (2001) showed that visual categories of stimuli can be classified based on neural activity, distributed and not clustered in small areas of the ventral temporal lobe. Subsequently, the feasibility of decoding has been explored using a variety of machine learning methods. Typically, these include various types of classifiers such as the logistic regressions, the Support Vector Machine, and the Gaussian Naive Bayes.

More recently, with the increasing interest in deep learning, studies applying non-linear multi-layer network models to decoding have been reported (Koyamada et al., 2015; Gao et al., 2019b; Thomas et al., 2019). Deep learning has the advantage of being able to simultaneously learn end-to-end, overcoming the previous faults of multi-step learning processes; previously, the classifier was learned after extracting brain regions as features, but it has now become possible to perform feature extraction and classifier learning from the whole brain at once (Wang et al., 2020). However, there remain some problems, such as the difficulty in applying deep learning to high-dimensional data and small sample size conditions (Cho et al., 2016; Yang et al., 2017).

In cognitive neuroimaging research, there tends to be a paucity of data due to experimental costs in terms of participant selection or session length, due to the complex demands of such research. When using machine learning for data analysis in sporadic experiments of this type, low statistical power and large errors in the evaluation of predictive accuracy often result. There is no clear solution to this issue, since it is important to exclude any unavoidable information leakage from a within-subject analysis. This is a crucial issue, especially when applied to a clinical context (Varoquaux et al., 2017; Varoquaux, 2018; Cearns et al., 2019).

In this study, in using a small-sized neurocognitive dataset, several cross-validation methods with different split units were used to evaluate the relative fit of different models. The models were used to analyze the results of a neurolinguistic experiment, from which a multi-site large-scale dataset is unlikely to be produced. In detail, we adopted a complicated task design for the experiment (conceptual association involving language switch), with an idiosyncratic subject group (early bilinguals familiar with two heterogeneous orthographic systems). This problem setting is particularly problematic for deep learning models because of the high-dimensional and small sample size dataset. At this point, we also identified the best method to adjust for and minimize information leakage to obtain desirable performance in the presence of a small-sized neurocognitive dataset.

\section{METHODS}

This study was performed in accordance with the Declaration of Helsinki and was approved by the Ethics Committee of the Tokyo Institute of Technology (approval number: B13001). Written informed consent was obtained from all subjects before participation. The details of the experiment are described in the Supplementary Material.

\section{Datasets}

Five Korean-Chinese early bilinguals participated in the functional magnetic resonance imaging (fMRI) experiments, which involved six repeated runs of a total of 20 mammal or 20 tool object images with name captions given in either Korean or Chinese, depending on the run numbers. The dataset consisted of 1,200 trials ( 6 runs $\times 40$ items for each subject; 600 trials for each class) produced by a rapid event-related design with stimulus randomization. For each trial, response data were obtained by using boxcars for 5-8 s after the stimulus onset (Akama et al., 2012); hence, there were four boxcars for which the magnitudes were averaged to generate data in each trial (except for one classifier described below). The target of the group-level MVPA was focused on the discrimination of the conceptual categories ("mammal" versus "tool"), although the language difference could result in a small degree of interference.

Using SPM8 (Friston et al., 1994), we performed a series of pre-processing steps including head movement correction, superimposing anatomical images, gray matter segmentation, conversion to Montreal Neurological Institute (MNI) coordinates, and resolution correction, after which a gray mask was applied using Nipy (Millman and Brett, 2007). Furthermore, each volume was cropped to exclude areas that were not part of the brain before $\mathrm{z}$-scoring the entire image.

\section{Classifiers}

Based on previous studies, we used four classifiers: penalized logistic regression (PLR), support vector machine (SVM), multichannel 2D convolutional neural network (M2DCNN), and $3 \mathrm{D}$ convolutional neural network (3DCNN). The codes for PLR and SVM were implemented using the Python package scikitlearn (Pedregosa et al., 2011), while those for M2DCNN and 3DCNN employed Pytorch (Paszke et al., 2019; both available at: https://github.com/sn0422j/mt_deep).

The PLR (L2 norm) and SVM (Linear SVM) were used, respectively, as the most popular classifiers. Regularization parameters were optimized with nested cross-validation (Nested$\mathrm{CV}$ ); for the activity vector, the boxcars were averaged, and 500 voxels were selected by analysis of variance (ANOVA).

For the M2DCNN model, we referred to the work of $\mathrm{Hu}$ et al. (2019), which meant that the model consisted of three twodimensional convolutional layers corresponding to the axes of three orthogonal planes, a merge layer that concatenates features, and a fully connected layer for classification. Figure 1A shows the architecture of this model. A Mish function (Misra, 2019) was used for the activation function to prevent overfitting. To train our model, we used cross-entropy as a loss function and Adam [learning rate $=0.001$, beta $=(0.9,0.999)]$ for optimization; 300 epochs were performed with exponential learning rate decay. The average images of the boxcars were used as the input to the model.

The 3DCNN model was based on the report of Wang et al. (2020; Figure 1B) to capture local spatiotemporal changes by applying three-dimensional convolutional filters over a time series. This training configuration was the same as the M2DCNN model, and the boxcars were used as the input to the model. It should be noted that the $3 \mathrm{DCNN}$ model allowed us to input 


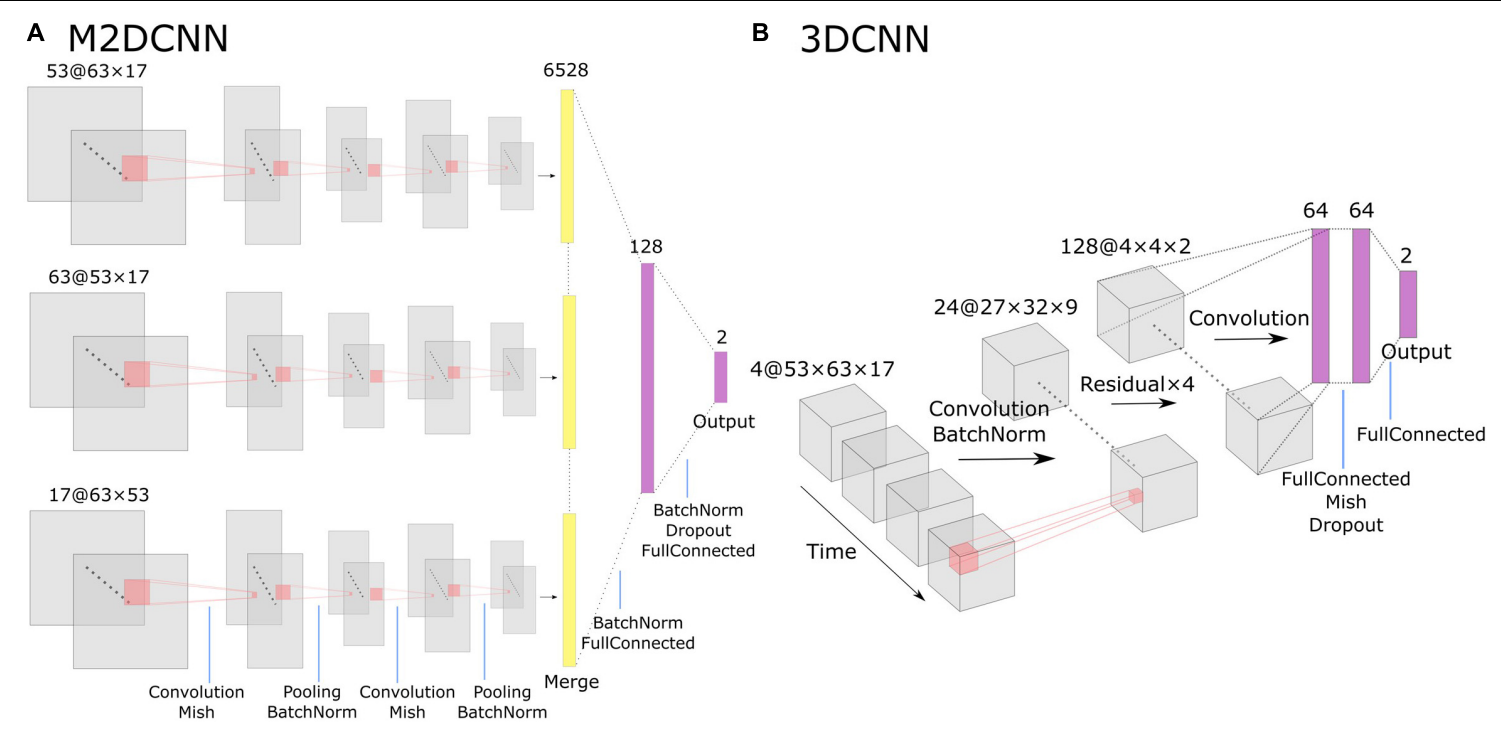

FIGURE 1 | The architecture of Two deep learning models: (A) the multichannel 2D convolutional neural network (M2DCNN) model and (B) the 3D convolutional neural network (3DCNN) model. Dropout rate of the fully connected layer was set to 0.5 in both (A) and (B).

without averaging the magnitudes of the critical boxcars as a single $4 \mathrm{D}$ data.

\section{Evaluation of Accuracy}

With a view to comparing the effectiveness of the following three cross-validation (CV) methods, we performed a five-fold CV for each method to calculate the classification accuracy of the test set split out from the small boxcar data: leave-one-subject-out CV, session shuffle split, and sample shuffle split. Note that for these $\mathrm{CV}$ methods, the data for the folds were subtracted from the six runs in each experiment, since we did not leave out any run(s) as a unit in this modeling.

When using the leave-one-subject-out $\mathrm{CV}$ as a splitting strategy, each subject was assigned a particular fold pattern so that only one individual's data was included in each test set (abbreviated hereafter as Test) and another one in the validation set (abbreviated as Valid) at every CV step (Figure 2A). Hence, each fold contained three subjects as providers of a training set (Train for short), one subject for the Valid, and another one for the Test.

In the session shuffle split, a fold was created in a run-byrun manner, regardless of subject identification, and by selecting $20 \%$ of the trials (taken as blocks) included in each run as Test or Valid at random (Figure 2B). The proportion of the numbers of data randomly assigned to the Train, Valid, and Test sets was identical throughout all folds (3:1:1). In other words, in each fold we had 18 runs for Train, 6 runs for Valid, and 6 runs for Test.

In the sample shuffle split method, a fold was set in a trialby-trial manner, without considering data attribution to subject and runs, and by randomly selecting 144 trials for Train, 48 trials for Valid, and the remaining 48 trials for Test (Figure 2C). The proportion of the three subsets was kept at 3:1:1 as was the case with the session shuffle split method.
Additionally, we performed a permutation test in which labels were randomly re-labeled 100 times, to calculate the chance level for each cross-validation method. With regards to the classifier, the PLR with the above settings was used as the baseline. We calculated the $p$ value for each combination of classifiers and $\mathrm{CV}$ methods using the Wilcoxon rank-sum test. The statistical analyses were conducted using Scipy.stats Version 1.4.1. A p value of less than 0.005 was the threshold for statistical significance.

\section{RESULTS}

The accuracy and $p$ values for the three cross-validations and the four classifiers are shown in Figure 3 and Table 1. PLR, SVM, and M2DCNN elicited significantly higher accuracy ( $p$ value $<0.0005)$ than the chance level for the session shuffle split and sample shuffle split. 3DCNN recorded almost the same accuracy as the chance level.

In general, the accuracy of the classifier was improved with, in ascending order, leave-one-subject-out CV, session shuffle split, and sample shuffle split. In the leave-one-subject-out $\mathrm{CV}$, the best precision rate $(0.511)$ was obtained with the PLR classifier, but this was not significant ( $p$ value $>0.005$ ). In the session shuffle split and sample shuffle split, the best precision rate $(0.640$, 0.751 , respectively) was obtained with the M2DCNN classifier. We regarded the value of 0.640 using session shuffle split as the authentic accuracy, which is discussed below.

\section{DISCUSSION}

\section{Comparison of Methods}

In this section, we review the properties of all of the split methods, respectively, and then assess the performance and structure of 


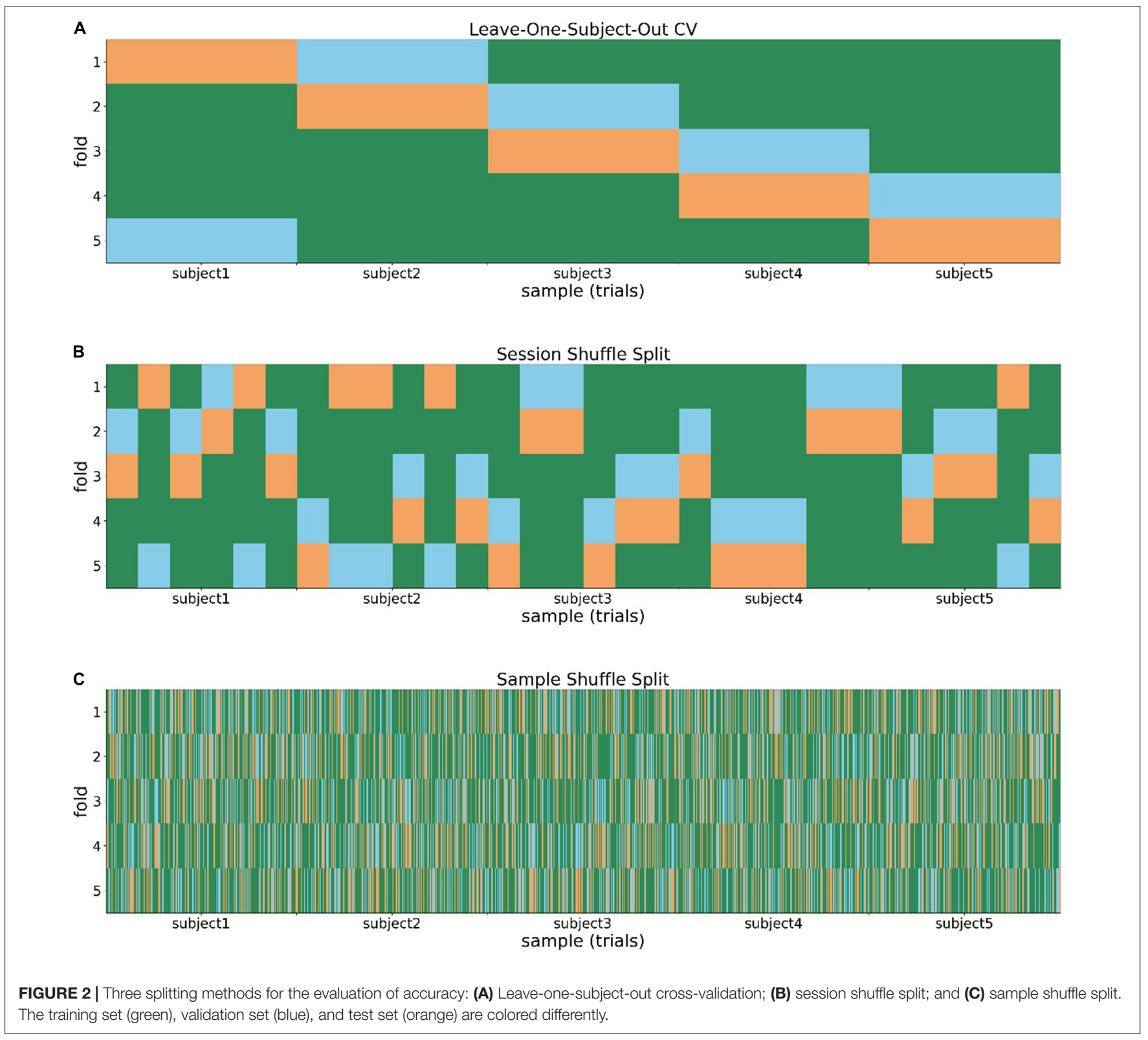

several deep learning models. Each CV method has a different data distribution within each of the splits. For the leave-onesubject-out CV, the breakdown of a fold composed of Train, Valid, and Test was limited to 3,1 , and 1 subject(s), respectively. Thus, we assume that the classifiers would be insufficient to achieve good generalization performance in the classification of further unknown subjects. The data were under-sampled from a large population of subjects, since the individual variability between subjects should be significantly larger than withinsubject fluctuations in terms of functional activity (Miller et al., 2009). As a result, statistical machine learning methods were far from a good fit.

When it comes to the session shuffle split and sample shuffle split methods, for which within-subject leakage was unpreventable, statistical machine learning was likely to be successful by reducing the effect of individual functional differences. Further improvement in the accuracy of the sample shuffle split may be dependent on the leakage caused in a time series due to the higher similarity of functional activity within runs than between runs (Varoquaux et al., 2017; Varoquaux, 2018). Moreover, it should be considered that two types of session-wise stimuli were provided to each subject with orthographic variability by language switch, which might have had a significant impact on his/her task performance.

Individual functional differences have traditionally limited the application of classifiers; solutions addressing this include functional alignment (hyperalignment; Haxby et al., 2011), the use of large datasets (Varoquaux et al., 2017; Varoquaux, 2018), and some few-shot learning techniques like transfer learning in deep learning (Gao et al., 2019a,b; Wang et al., 2020), 


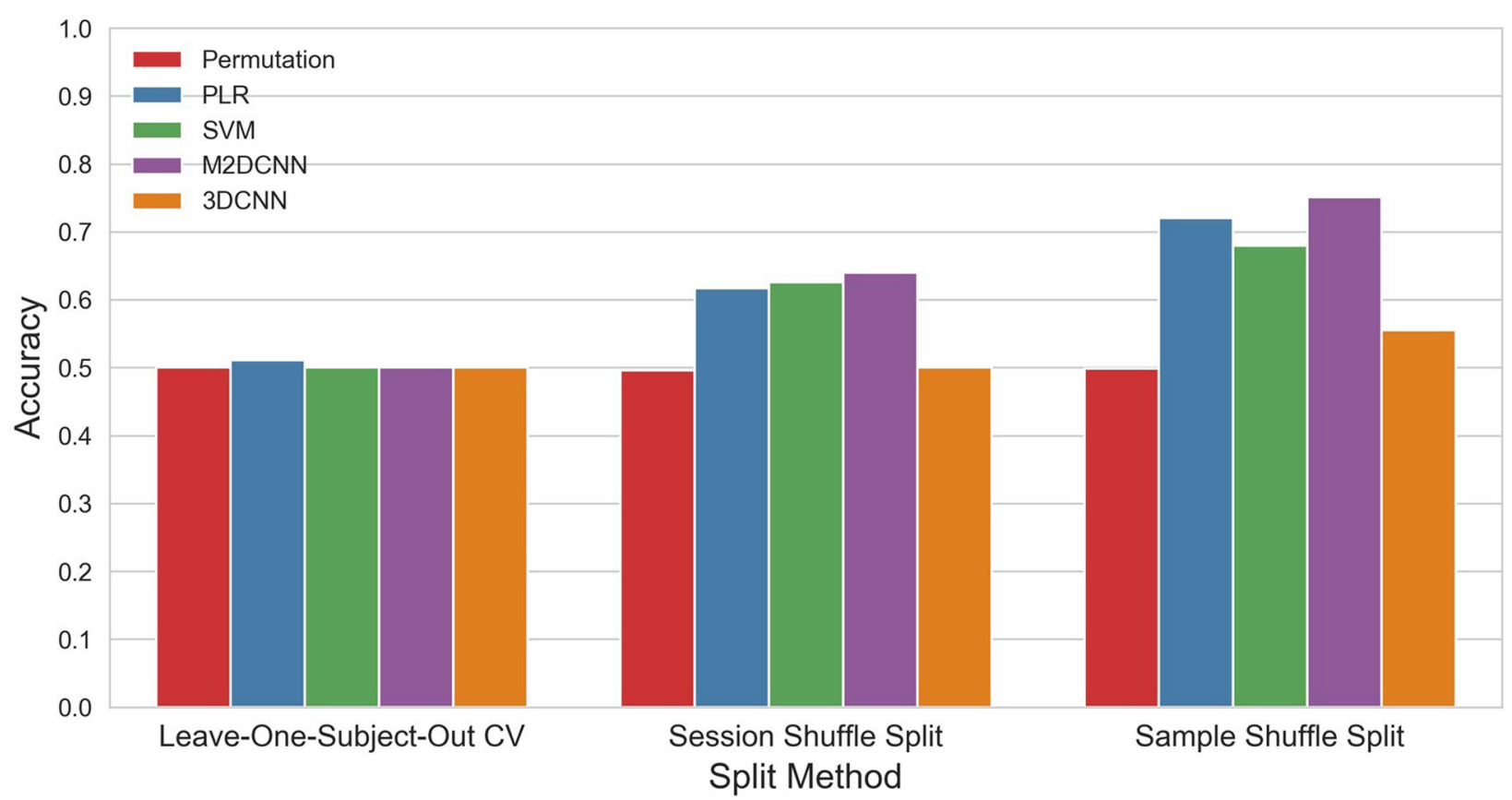

FIGURE 3 | The mean accuracy for each split method and classifier. The permutation accuracy represents the chance level for each split method. 3DCNN, three-dimensional convolutional neural network; M2DCNN, multichannel two-dimensional convolutional neural network; PLR, penalized logistic regression; SVM, support vector machine.

TABLE 1 | The mean accuracy and the associated $p$ value for each split method and classifier.

\begin{tabular}{llcc}
\hline Split method & Training method & Accuracy & p value \\
\hline Leave-One-Subject-Out CV & PLR & $\mathbf{0 . 5 1 1}$ & 0.1529 \\
& SVM & 0.500 & 0.9760 \\
& M2DCNN & 0.500 & 0.9700 \\
SDCNN & 0.500 & 0.9700 \\
Session Shuffle Split & PLR & 0.617 & $0.0002^{\text {** }}$ \\
& SVM & 0.626 & $0.0002^{\text {** }}$ \\
& M2DCNN & $\mathbf{0 . 6 4 0}$ & $0.0002^{\text {** }}$ \\
Sample Shuffle Split & 3DCNN & 0.500 & 0.9101 \\
& PLR & 0.720 & $0.0002^{\text {** }}$ \\
& SVM & 0.680 & $0.0002^{\text {** }}$ \\
& M2DCNN & $\mathbf{0 . 7 5 1}$ & $0.0002^{\text {** }}$ \\
& 3DCNN & 0.555 & $0.0017^{\star}$
\end{tabular}

A single asterisk $\left({ }^{*}\right)$ indicates $p<0.005$; double asterisks $\left(^{* *}\right)$ indicate $p<0.0005$. $\mathrm{CV}$, cross-validation. The highest accuracy in each split method is highlighted in bold.

transfer learning in shared response modeling (Zhang et al., 2018; Yousefnezhad et al., 2020), and meta-learning. It is difficult, however, to use these methods for deep learning with a limited sample size and a unique experimental condition. Therefore, leaking information to some extent as referenceable prior knowledge and discussing end-to-end models appears to be one of the better solutions to address this issue. The session shuffle split model appears to be the best way to evaluate the accuracy of the models in this case, since the individual functional differences are referenceable without being affected by the timeseries correlation.

The M2DCNN model, which achieved the highest accuracy with little information leakage, was evaluated using gradients to locate what the model learned for classification. This analysis resulted in consistency with prior research describing similar experimental tasks (see Supplementary Material for analytical details). In this regard, a deep learning end-to-end model could detect category-specific responses that are common to the subjects.

The unexpectedly poor efficiency of the $3 \mathrm{DCNN}$ model for the present analysis is worthy of discussion. Prior studies that applied the 3DCNN model to task fMRI (Hu et al., 2019; Wang et al., 2020) showed high accuracy in block designs, with sustained and homogeneous task characteristics. The rapid event-related design that we employed in our experiment might promote greater variability within the time series. Given this, a model that explicitly incorporates time series information, such as long short-term memory (LSTM), may fit better (Thomas et al., 2019) for a checkered experiment session.

\section{Limitations and Future Directions}

In this section, we provide some limitations of this study and discuss the best method to adjust the information leakage level. There are some limitations to this study. The accuracy reported in this study is not an indicator of the generalizable performance of the entire subject population, due to the leakage of information. Here, we define information leakage as the phenomenon where the i.i.d. split units for each split strategy have dependence as 
a consequence of the structured property of data distribution. There are several levels of information leakage, which should be separated out in terms of legitimacy (Kaufman et al., 2012). Based on this idea, we propose readily attributing levels as "heavy" or "light" for those actions.

In the fMRI decoding framework, heavy leakage has been considered to be so serious that it affects the authenticity of accuracy indicators, such as that seen in supervised feature selection prior to splitting or hyperparameter optimization with Test data (Kaufman et al., 2012). In contrast, light leakage is likely to occur when the Train and Test data are not completely independent, with their indirect and hidden relationship being difficult to scrutinize; its impact is generally taken as small but complex and essentially unknown.

In this study, we presented an example of training a complex model by allowing light information leakage. For group analysis, the sample shuffle split method ignored the leakage likely to be caused in a time series and hypothesized the independence of trials within runs. We believe that under this condition, the rate of 0.651 obtained by the session shuffle split and the M2DCNN classifier was the authentic limit of classification accuracy in this study. Beyond this scope, some results of multivariate analysis based on heavy leakage might be considered to work entirely outside the context of machine learning; for example, an adaptive reuse of them is possible, such as that seen with a brain semantic map reflecting the representational similarity of concepts. However, open questions remain unanswered in relation to the utility of such rich information handling.

In regards to the underestimation of cross-validation loss, our research indicated a need to demonstrate how we could control the data independence and support the significance of the indicator in a non-parametric way; for example, by using a permutation test (Varoquaux et al., 2017; Varoquaux, 2018). However, when investigating cognitive processes specific to a narrow population as in the case of this study, it is important to model within-subject variability by taking more data, even with fewer subjects, and reducing within-subject errors (Smith and Little, 2018). Future studies are required to develop and train a more reliable classifier for each subject and to stably as well as precisely detect consistent shared effects across subjects with higher statistical power.

\section{CONCLUSION}

In this study, we examined the application of complex models for the decoding of fMRI under the constrained condition of a small

\section{REFERENCES}

Akama, H., Murphy, B., Na, L., Shimizu, Y., and Poesio, M. (2012). Decoding semantics across fMRI sessions with different stimulus modalities: a practical MVPA study. Front. Neuroinform. 6:24. doi: 10.3389/fninf.2012.000 24

Cearns, M., Hahn, T., and Baune, B. T. (2019). Recommendations and future directions for supervised machine learning in psychiatry. Transl. Psychiatry 9:271. doi: 10.1038/s41398-019-0607-2 sample size in a unique cognitive experiment. It was shown that even when data bias was caused by functional variability across subjects, in spite of greatly limited performance of the classifiers, the complex model could be successfully applied by taking a moderate split to control information leakage. This might be a key to success in deep learning for overcoming a paucity of fMRI data. In this study, we have discussed the tolerability of within-subject or within-session information leakage, of which the impact was generally considered to be small but complex and essentially unknown; this requires clarification in future studies.

\section{DATA AVAILABILITY STATEMENT}

The raw data supporting the conclusions of this article will be made available by the authors, without undue reservation.

\section{ETHICS STATEMENT}

The studies involving human participants were reviewed and approved by the Ethics Committee of the Tokyo Institute of Technology (approval number: B13001). The patients/participants provided their written informed consent to participate in this study.

\section{AUTHOR CONTRIBUTIONS}

SY and HA: conceptualization, investigation, methodology, and writing. SY, ML, and HA: data curation and analysis. HA: supervision. All authors contributed to the article and approved the submitted version.

\section{ACKNOWLEDGMENTS}

We would like to thank Y. Minowa for useful discussions. We would also like to thank Editage (www.editage.com) for English language editing. Finally, we are grateful to the subjects for participating in the experiment.

\section{SUPPLEMENTARY MATERIAL}

The Supplementary Material for this article can be found online at: https://www.frontiersin.org/articles/10.3389/fninf. 2021.577451/full\#supplementary-material

Cho, J., Lee, K., Shin, E., Choy, G., and Do, S. (2016). How much data is needed to train a medical image deep learning system to achieve necessary high accuracy? arXiv [Preprint]. Available online at: https://arxiv.org/abs/1511.06348 (accessed April 25, 2020).

Cohen, J. D., Daw, N., Engelhardt, B., Hasson, U., Li, K., Niv, Y., et al. (2017). Computational approaches to fMRI analysis. Nat. Neurosci. 20, 304-313. doi: 10.1038/nn.4499

Friston, K. J., Holmes, A. P., Worsley, K. J., Poline, J. P., Frith, C. D., and Frackowiak, R. S. J. (1994). Statistical parametric maps in functional imaging: 
a general linear approach. Hum. Brain Mapp. 2, 189-210. doi: 10.1002/hbm. 460020402

Gao, Y., Zhang, Y., Cao, Z., Guo, X., and Zhang, J. (2019a). Decoding brain states from fMRI signals by using unsupervised domain adaptation. IEEE J. Biomed. Health Inform. 24, 1677-1685. doi: 10.1109/JBHI.2019.2940695

Gao, Y., Zhang, Y., Wang, H., Guo, X., and Zhang, J. (2019b). Decoding behavior tasks from brain activity using deep transfer learning. IEEE Access 7, 4322243232. doi: 10.1109/ACCESS.2019.2907040

Haxby, J. V., Gobbini, M. I., Furey, M. L., Ishai, A., Schouten, J. L., and Pietrini, P. (2001). Distributed and overlapping representations of faces and objects in ventral temporal cortex. Science 293, 2425-2430. doi: 10.1126/science.1063736

Haxby, J. V., Guntupalli, J. S., Connolly, A. C., Halchenko, Y. O., Conroy, B. R., Gobbini, M. I., et al. (2011). A common, high-dimensional model of the representational space in human ventral temporal cortex. Neuron 72, 404-416. doi: 10.1016/j.neuron.2011.08.026

Hu, J., Kuang, Y., Liao, B., Cao, L., Dong, S., and Li, P. (2019). A multichannel 2D convolutional neural network model for task-evoked fMRI data classification. Comput. Intell. Neurosci. 2019:5065214. doi: 10.1155/2019/5065214

Kaufman, S., Rosset, S., Perlich, C., and Stitelman, O. (2012). Leakage in data mining: formulation, detection, and avoidance. ACM Trans. Knowl. Discov. Data 6:15. doi: 10.1145/2382577.2382579

Koyamada, S., Shikauchi, Y., Nakae, K., Koyama, M., and Ishii, S. (2015). Deep learning of fMRI big data: a novel approach to subject-transfer decoding. arXiv [Preprint]. Available online at: https://arxiv.org/abs/1502.00093 (accessed April $25,2020)$.

Miller, M. B., Donovan, C. L., Van Horn, J. D., German, E., Sokol-Hessner, P., and Wolford, G. L. (2009). Unique and persistent individual patterns of brain activity across different memory retrieval tasks. Neuroimage 48, 625-635. doi: 10.1016/j.neuroimage.2009.06.033

Millman, K. J., and Brett, M. (2007). Analysis of functional magnetic resonance imaging in Python. Comput. Sci. Eng. 9, 52-55. doi: 10.1109/MCSE.2007.46

Misra, D. (2019). Mish: a self regularized non-monotonic neural activation function. arXiv [Preprint]. Available online at: https://arxiv.org/abs/1908.08681 (accessed April 26, 2020).

Paszke, A., Gross, S., Massa, F., Lerer, A., Bradbury, J., Chanan, G., et al. (2019). "PyTorch: an imperative style, high-performance deep learning library," in Advances in Neural Information Processing Systems, eds H. Wallach, H. Larochelle, A. Beygelzimer, F. Alché-Buc, E. Fox, and R. Garnett (Red Hook, NY: Curran Associates, Inc), 8024-8035.
Pedregosa, F., Varoquaux, G., Gramfort, A., Michel, V., Thirion, B., Grisel, O., et al. (2011). Scikit-learn: machine learning in Python. J. Mach. Learn. Res. 12, 2825-2830.

Smith, P. L., and Little, D. R. (2018). Small is beautiful: in defense of the small-N design. Psychon. Bull. Rev. 25, 2083-2101. doi: 10.3758/s13423-018-1451-8

Thomas, A. W., Heekeren, H. R., Müller, K. R., and Samek, W. (2019). Analyzing neuroimaging data through recurrent deep learning models. arXiv [Preprint]. Available online at: https://arxiv.org/abs/1810.09945 (accessed April 25, 2020).

Varoquaux, G. (2018). Cross-validation failure: small sample sizes lead to large error bars. Neuroimage 180, 68-77. doi: 10.1016/j.neuroimage.2017.06.061

Varoquaux, G., Raamana, P. R., Engemann, D. A., Hoyos-Idrobo, A., Schwartz, Y., and Thirion, B. (2017). Assessing and tuning brain decoders: cross-validation, caveats, and guidelines. Neuroimage 145, 166-179. doi: 10.1016/j.neuroimage. 2016.10.038

Wang, X., Liang, X., Jiang, Z., Nguchu, B. A., Zhou, Y., Wang, Y., et al. (2020). Decoding and mapping task states of the human brain via deep learning. Hum. Brain Mapp. 41, 1505-1519. doi: 10.1002/hbm.24891

Yang, Q., Liu, B., Wei, Y., and Zhang, Y. (2017). "Deep neural networks for high dimension, low sample size data," in Proceedings of the 26th International Joint Conference on Artificial Intelligence (IJCAI'17 (Palo Alto, CA: AAAI Press), 2287-2293.

Yousefnezhad, M., Selvitella, A., Zhang, D., Greenshaw, A. J., and Greiner, R. (2020). Shared space transfer learning for analyzing multi-site fMRI data. arXiv [Preprint]. Available online at: http://arxiv.org/abs/2010.15594 (accessed January 20, 2021).

Zhang, H., Chen, P.-H., and Ramadge, P. (2018). "Transfer Learning on fMRI Datasets," in Proceedings of the Twenty-First International Conference on Artificial Intelligence and Statistics, Playa Blanca, 595-603.

Conflict of Interest: The authors declare that the research was conducted in the absence of any commercial or financial relationships that could be construed as a potential conflict of interest.

Copyright (c) 2021 Yotsutsuji, Lei and Akama. This is an open-access article distributed under the terms of the Creative Commons Attribution License (CC BY). The use, distribution or reproduction in other forums is permitted, provided the original author(s) and the copyright owner(s) are credited and that the original publication in this journal is cited, in accordance with accepted academic practice. No use, distribution or reproduction is permitted which does not comply with these terms. 